\title{
Uniaxial Strain Induced Critical Current Degradation of Ag-Sheathed Bi-2212 Round Wire
}

\author{
Chao Dai, Jinggang Qin ${ }^{(0)}$, Bo Liu, Peihang Liu, Yu Wu, Arend Nijhuis, Chao Zhou, Chenshan Li, Qingbin Hao, \\ and Sheng Liu
}

\begin{abstract}
The critical current degradation of Bi-2212 Ag-sheathed round wire subjected to uniaxial strain was studied at $4.2 \mathrm{~K}$ in $14 \mathrm{~T}$ background field. The strains applied on the sample are both tension and compression. The additional tensile strain caused by the difference in thermal expansion between the Bi-2212 round wire and $\mathrm{Ti}-6 \mathrm{Al}-\mathbf{4 V}$ alloy spring was calculated. The results showed that a drastic degradation of the critical current occurred when the intrinsic strain exceeded around $0.5 \%$ in tensile direction. For the compressive strain, the degradation of critical current was almost linear but more gradual than tensile strain. The intention is to use these results as a basis for $\mathrm{Bi}-2212$ conductor and superconducting coil design.
\end{abstract}

Index Terms-Axial strain, Bi-2212 round wires, irreversible critical current.

\section{INTRODUCTION}

B I-2212 high- $T_{\mathrm{c}}$ superconductor is a promising superconducting material, which could be used in high magnetic field devices, such as nuclear fusion reactors [1], [2]; accelerator and nuclear magnetic resonance magnets (NMR), due to its very high upper critical field. More importantly, it can be made in round wire, which means it can be a suitable candidate for cable in conduit conductor (CICC).

$\mathrm{Bi}-2212$ round wire is sensitive to strain [3]-[6]. As a kind of cuprate superconductor, the Bi-2212 phase is brittle like ceramic, and its $\mathrm{Ag} / \mathrm{Ag}-\mathrm{Mg}$ sheath has low strength. Under operating conditions, electromagnetic force and thermal stress can initiate cracks on it easily, which would result in critical current $\left(I_{c}\right)$ degradation.

Currently, a CICC is under development for the next generation fusion reactor at ASIPP (the Institute of Plasma Physics, CAS), The CICC design is based on $\mathrm{Bi}-2212$ round wire developed by Northwest Institute for Non-Ferrous Metal Research

Manuscript received August 29, 2017; accepted December 18, 2017. Date of publication December 25, 2017; date of current version January 17, 2018. This work was supported by National Magnetic Confinement Fusion Science Program under Grant 2014GB105001. (Corresponding author: Jinggang Qin.)

C. Dai, J. Qin, B. Liu, P. Liu, and Y. Wu are with the Institute of Plasma Physics, Hefei 230031, China (e-mail: qinjg@ipp.ac.cn).

A. Nijhuis and C. Zhou are with the Department of Energy, Materials, and Systems, Faculty of Science and Technology, University of Twente, Enschede 7500AE, Netherlands.

C. Li and Q. Hao are with the Northwest Institute for Non-Ferrous Metal Research, Xi'an 710016, China.

S. Liu is with the China International Nuclear Fusion Energy Program Execution Center, Beijing 100862, China.

Color versions of one or more of the figures in this paper are available online at http://ieeexplore.ieee.org.

Digital Object Identifier 10.1109/TASC.2017.2787133

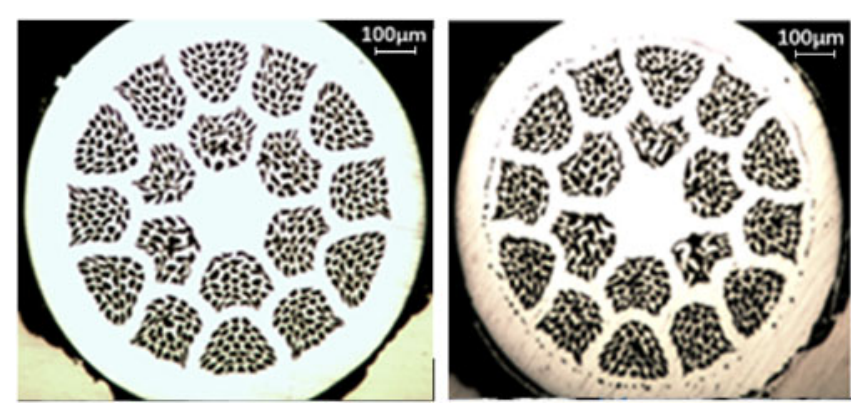

Fig. 1. The cross-sectional micrographs of Bi2212 RW pre-reaction (left) and post-reaction (right)

TABLE I

PARAMETERS OF THE TESTED BI2212 WIRE

\begin{tabular}{lc}
\hline \hline Parameters & Ag-alloy sheathed Bi-2212 \\
\hline Diameter & $1.0 \mathrm{~mm}$ \\
Filament configuration & $19 \times 18$ \\
$\mathrm{Ag} / \mathrm{Mg}: \mathrm{Ag}: \mathrm{Bi} 2212$ & $1.8: 1: 0.9$ \\
$I_{c}$ at $0 \mathrm{~T}, 4.2 \mathrm{~K}$ & about $400 \mathrm{~A}$ \\
$I_{c}$ at $12 \mathrm{~T}, 4.2 \mathrm{~K}$ & about $146 \mathrm{~A}$ \\
\hline \hline
\end{tabular}

(NIN). Since this kind of Bi-2212 round wire is newly developed in China, so it's necessary to study its performances before application. The strain dependence critical performance study is important for predicting the degradation of conductor under electromagnetic load. In CICC cable design, the strain dependence critical performance could be a significant factor for determining the key parameters of cable, such as twist pitch and void fraction. In this work, the strain applied on the Bi-2212 round wire was from $-0.6 \%$ to $+0.3 \%$ with the $\mathrm{U}$-spring device [5], at a temperature of $4.2 \mathrm{~K}$ and an applied magnetic field of $14 \mathrm{~T}$. The test results are presented and discussed in this paper.

\section{EXPERIMENTAL SETUP}

\section{A. Sample Preparation}

The Bi-2212 round wires were fabricated by Northwest Institute for Non-Ferrous Metal Research (NIN) using the powderin-tube (PIT) process. The cross sections of the round wire are shown in Fig. 1, The basic performance and filament configuration of this $\mathrm{Bi}-2212$ round wire were listed in Table I. The heat treatment process was also performed at Northwest Institute for Non-Ferrous Metal Research, at a maximum melting temperature $\mathrm{T}_{\max }=890^{\circ} \mathrm{C}$ for 2 hours. The two ends of each 


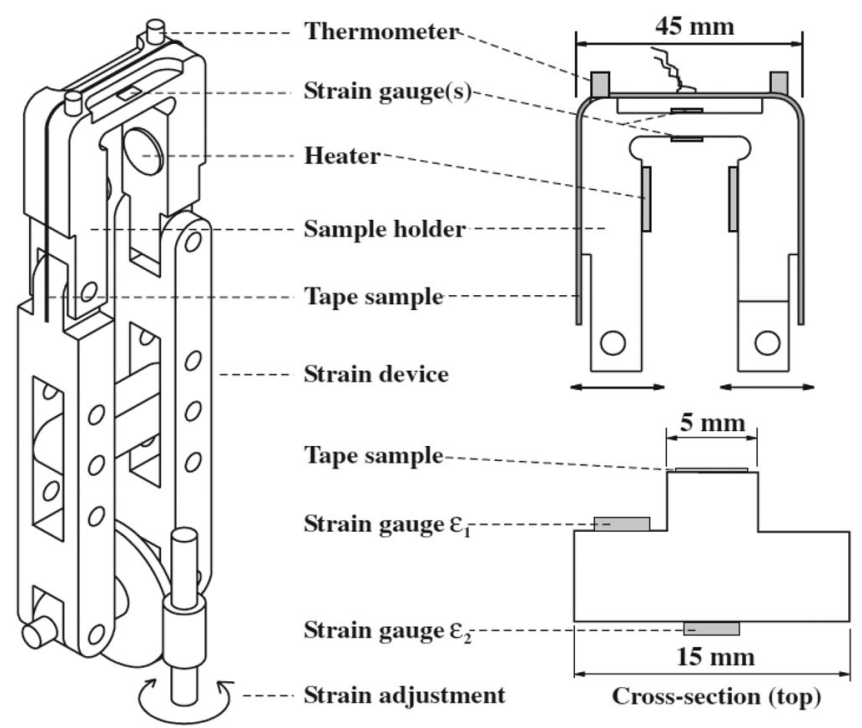

Fig. 2. The structure and operating principle of $\mathrm{U}$-spring device.

sample should be flattened, otherwise the melting Bi-2212 powder would leak from the matrix through the ends.

\section{B. Device and Experimental Procedure}

The experiments were done at University of Twente with the U-spring device. Tensile and compression axial strains can be applied on the sample at different temperature and magnetic field $I_{c}(B, T, \varepsilon)$ with this device. In experiment, the sample wire/tape is soldered or glued to a U-shape substrate. Strain is applied to the sample by bending the substrate. The bending force is transferred and adjusted by lever and worm, the operating principle is shown in Fig. 2 [8], [9]. U-spring device has advantage of short, straight sample testing. For Bi-2212 round wire, its heat treatment condition is harsh for many materials, short and straight sample is simplest for heat treatment and can minimize damage. The U-shape Ti-6Al-4V alloy spring has a wide elastic strain range between $1 \%$ and $-1 \%$. The U-spring device and the FEM calculated strain profiles of U-shape spring are shown in Fig. 3.

After the wire heat treatment, each straight sample was soldered on the homogeneous strain region of the U-shape spring. Two pairs of voltage taps are attached on the strand surface at the center of the tested sample, with the distance of 8 and $16 \mathrm{~mm}$, respectively. The distance from the outer voltage tap to current lead is $10 \mathrm{~mm}$. The outer voltage tap pair is used to check whether any entrance effect occurs [10]. The electric-field criterion is $E_{c}=100 \mu \mathrm{V} / \mathrm{m}$ and the n-value is determined between $10-100 \mu \mathrm{V} / \mathrm{m}$. The $I_{c}$ results were taken from the inner pair of voltage taps with a distance of $8 \mathrm{~mm}$.

Strain dependent critical current measurements were carried out in liquid helium under different temperatures and magnetic fields. At first two samples were tested to investigate the basic performance of this Bi-2212 round wire at $4.2 \mathrm{~K}$ and $20 \mathrm{~K}$ and various magnetic fields from $0-14 \mathrm{~T}$ without applied strain apart from the thermal contraction by the spring, the temperature variation is less than $10 \mathrm{mK}$ in the case of $4.2 \mathrm{~K}$ and $20 \mathrm{~K}$. Then

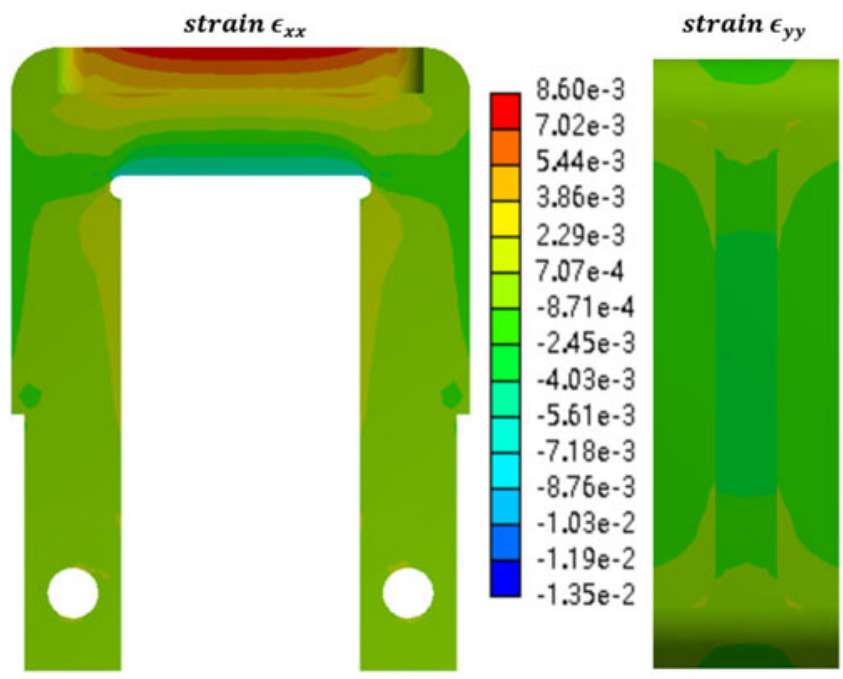

Fig. 3. FEM calculated strain profiles of the U-spring.

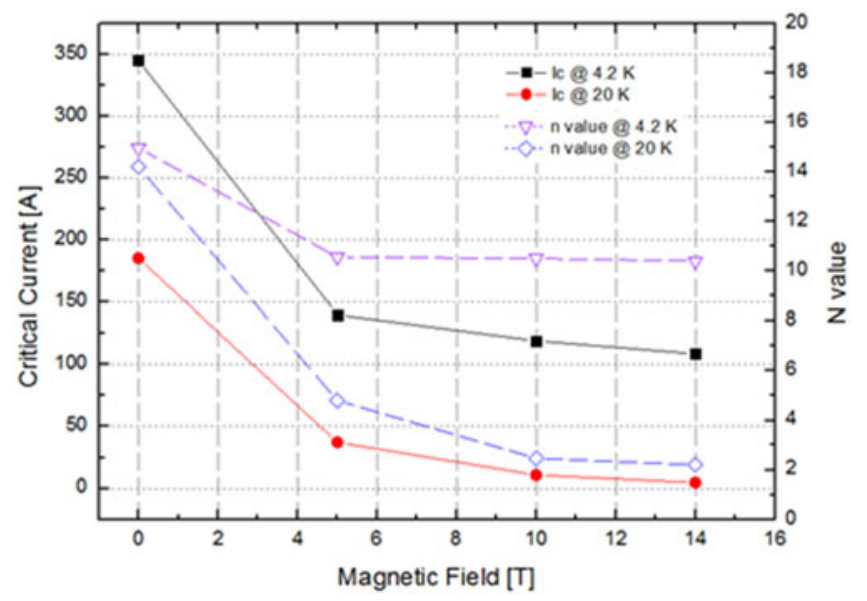

Fig. 4. The critical current and n-value versus magnetic field.

two samples were tested with tensile and compressive strains respectively at $4.2 \mathrm{~K}$ and $14 \mathrm{~T}$.

\section{Results AND Discussions}

The critical currents and n-values of the two samples were measured to investigate the basic performance of the Bi-2212 round wire at $4.2 \mathrm{~K}$ and $20 \mathrm{~K}$ with various magnetic fields, are plotted in Fig. 4.

It is shown that the $I_{c}$ degrades dramatically with increasing magnetic field when up to $5 \mathrm{~T}$. At higher magnetic field the degradation rate of the $I_{c}$ gets significantly less. The n-value shows the similar behavior as that of the $I_{c}$.

Since permanent $I_{c}$ degradation and crack formation can occur due to both tension and compression, two Bi-2212 samples were measured to investigate the strain dependence of critical current at $4.2 \mathrm{~K}$ and $14 \mathrm{~T}$ under tensile and compressive strain, respectively. One sample (S1) was measured under tensile axial strain. The result of sample S1 is shown in Fig. 5. The other sample (S2) was measured under compressive axial strain, as shown in Fig. 6. Because the tensile and compressive strains 


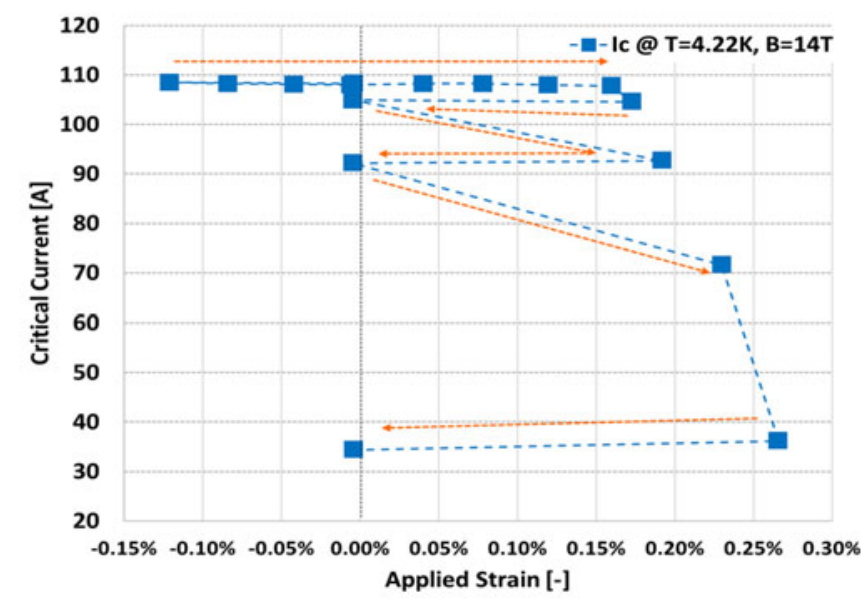

Fig. 5. The $I_{c}$ of $\mathrm{S} 1$ as a function of applied tensile strain at $4.2 \mathrm{~K}$ and $14 \mathrm{~T}$.

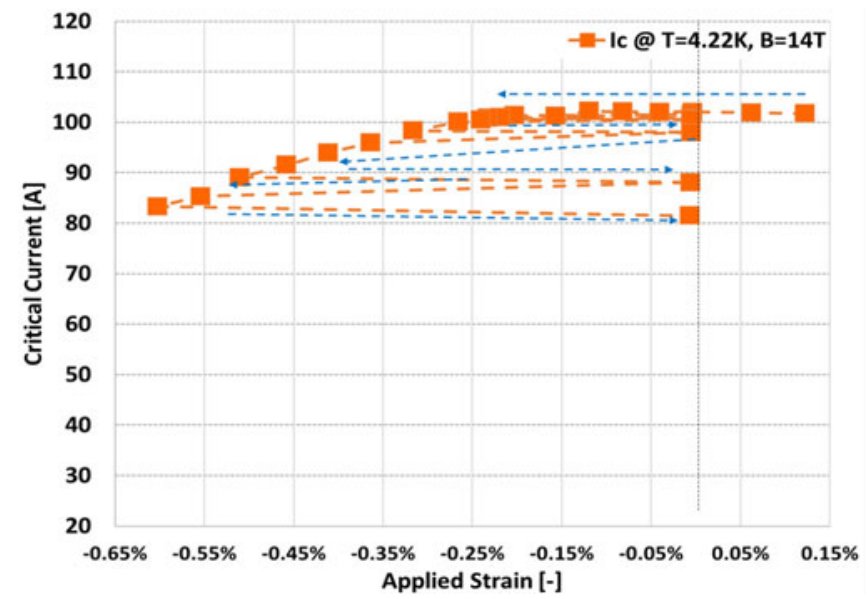

Fig. 6. The $I_{c}$ of S2as a function of applied compressive strain at $4.2 \mathrm{~K}$ and $14 \mathrm{~T}$.

were applied on two samples, there is several amperes difference in $I_{c}$ between two samples at zero applied strain. The two curves of the normalized $I_{c}$ versus applied compressive and tensile strain were put together for a good comparison in Fig. 7. The normalized $I_{c}$ equals to $I_{c} / I_{c 0}$, where $I_{c 0}$ is the critical current measured before applying strain on the sample.

The strain dependence of the critical current of Bi-2212 round wires is quite different from the quasi-parabolic behavior of niobium based low-temperature superconductors [11]-[13]. The irreversible reduction of $I_{c}$ occurred around $0.17 \%$ for applied tensile strain, and around $-0.22 \%$ for applied compressive strain. In this range of applied strain from $-0.2 \% \sim+0.15 \%$, the $I_{c}$ of the Bi-2212 wire isn't very sensitive to the strain.

But other experiment results [4], [5], [14], [21] indicate that the $I_{c}$ decreased very slowly by about $2 \%$ at an applied strain of $0.3 \% \sim 0.4 \%$ on the tension side, and on compressive side, $I_{c}$ dropped almost linearly and quickly all the way. This seems different from our results especially on compressive side. This difference is just caused by the different initial strains applied on the samples with different material of substrate (B. tenHaken's experiment was with brass spring and $\mathrm{N}$. Cheggour's experiment was with $\mathrm{Cu}-\mathrm{Be}$ spring). A previous study by Sugano et al.,

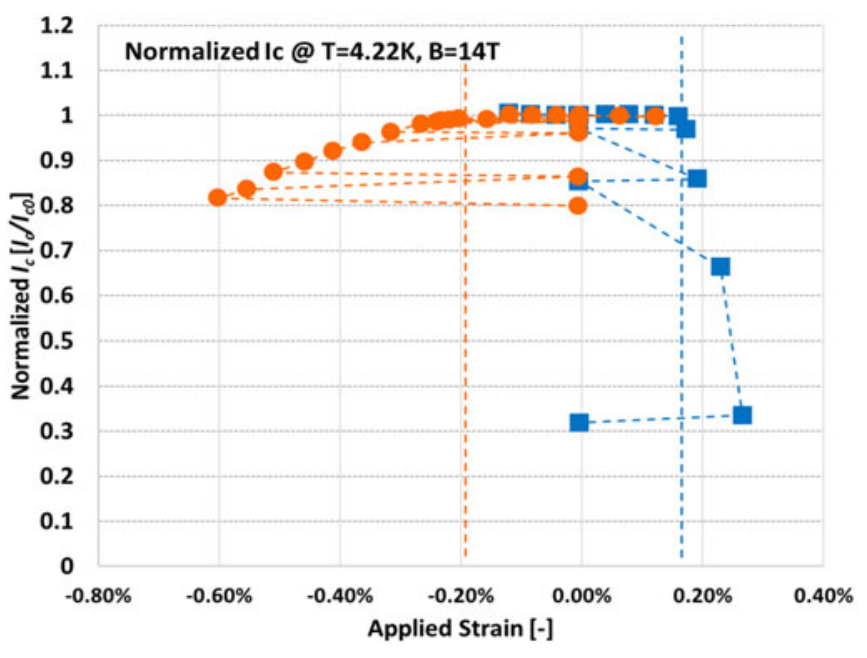

Fig. 7. Normalized $I_{c}$ of sample $\mathrm{S} 1$ and $\mathrm{S} 2$ as a function of applied strain at $4.2 \mathrm{~K}$ and $14 \mathrm{~T}$.

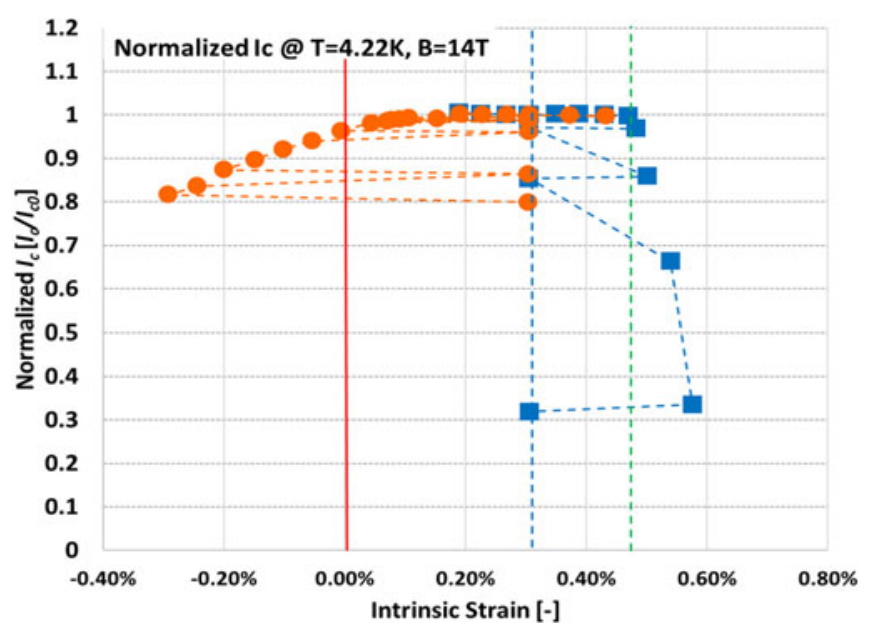

Fig. 8. Normalized $I_{c}$ as a function of Intrinsic strain at $4.2 \mathrm{~K}$ and $14 \mathrm{~T}$.

showed that the thermal expansion of $\mathrm{Cu}-\mathrm{Be}$ matches that of a Bi-2212 composite wire very well (to within $0.01 \%$ between $290 \mathrm{~K}$ and $5 \mathrm{~K}$ ) [15]. But if using Ti-6Al-4V as substrate could cause a $+0.31 \%$ tension strain on $\mathrm{Bi}-2212$ round wire when temperature cold down to $4.2 \mathrm{~K}$ from soldering temperature (about $460 \mathrm{~K}$ ) [16], [20]. After shifting the zero applied strain point by $-0.31 \%$ on the strain-axis, the normalized $I_{c}$ versus intrinsic strain is shown in Fig. 8. From Fig. 8, it can be found that the irreversible intrinsic strain is about $0.502 \%$ on the tension side. This value was reported to be $0.4 \%-0.6 \%$ of different samples in A. Godeke's paper [20]. In N. Cheggour's paper [21], the substrate material is $\mathrm{Cu}-\mathrm{Be}$, and the irreversible applied strain is around $0.4 \%$, which is similar to the intrinsic strain measured by Ti- $6 \mathrm{Al}-4 \mathrm{~V}$ substrate. The comparison indicates that $\mathrm{Cu}-\mathrm{Be}$ substrate is more appropriate to be the substrate material for $\mathrm{Bi}-2212$ wire strain test.

Generally, the drastic reduction of $I_{c}$ on tensile side is caused by cracks [3]-[5]. The $I_{c}$ versus wire strain and wire stress versus wire strain at $4.2 \mathrm{~K}$ was compared, which was measured before [17]. The results are shown in Fig. 9. From Fig. 9, It can 


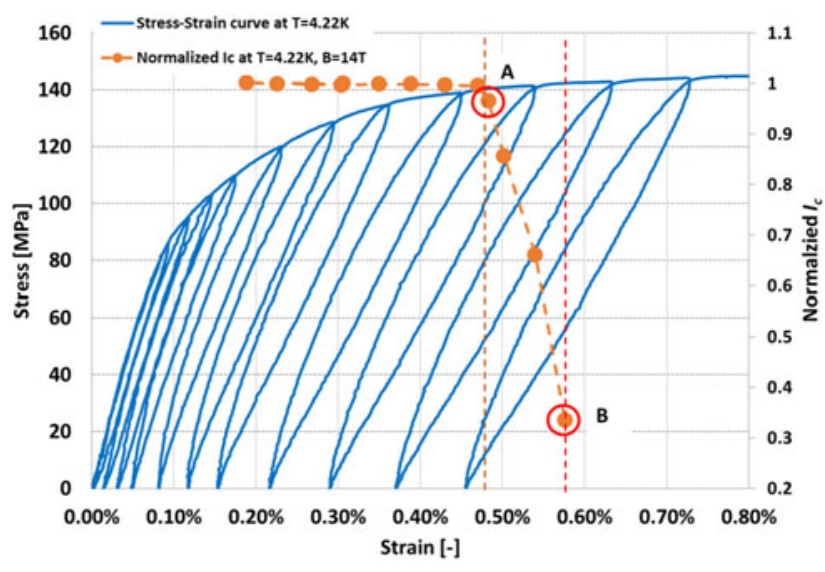

Fig. 9. Comparison of Bi-2212 wire stress and $I_{c}$ versus strain curves at $4.2 \mathrm{~K}$ and $14 \mathrm{~T}$.

be seen that the precipitous degradation of $I_{c}$ occurred when the wire strain was around $0.502 \%$ (point A). And a drastic degradation of $I_{c}$ occurred when the wire strain was around $0.576 \%$ (point B), at this strain, the $I_{c}$ decreased by almost $70 \%$, presumably indicating that a strong damage of the $\mathrm{Bi}$ 2212 filaments. On the other hand, at the strain about $0.5 \%$, the wire stress approached the plateau at this strain, which means the wire has entered plastic stage, this suggests that stress-strain measurements may provide a good indication of the strain and stress limit at which strong Bi-2212 filament damage occurs. These filaments breakage leads to the drastic degradation of $I_{c}$. This also happens to the other Bi-base high- $T_{c}$ material, such as the Bi-2223 tape [19].

For the compressive strain dependence of the critical current, the reduction of $I_{c}$ maybe caused by buckling [3]-[5]. This reduction is more gradual than tension, the $I_{c}$ decreased by $20 \%$ for a compressive strain of $-0.4 \%$ since cracks occur less easily under compressive strain.

\section{CONCLUSION}

The impact of applied axial strain to Bi-2212 Ag/Ag-Mg sheathed round wire was investigated in both compression and tensile direction. The critical current degraded as a function of both compressive and tensile strain side. A correction was applied for the additional $0.31 \%$ tensile strain on the samples caused by the difference of the thermal expansion coefficient between $\mathrm{Bi}-2212$ round wire and Ti-6Al-4V. No noticeable degradation of $I_{c}$ was observed for the intrinsic strain up to $0.502 \%$, while exceeding this irreversibility limit, a dramatic degradation occurred with increased strain. The $I_{c}$ decreases almost linearly for compressive strain, but more gradual than under tension. The reduction of $I_{c}$ on tension side agrees well with the yielding in the stress-strain characteristic. Further studies will be performed to verify whether this strain dependence of $I_{c}$ is intrinsic to $\mathrm{Bi}-2212$ round wire, a $\mathrm{Cu}-\mathrm{Be} \mathrm{U}$-spring will be made for further experiments. Then the morphology of cracks will be investigated.
On the other hand, we plan to investigate the strain dependence critical performance of $\mathrm{Bi}-2212$ round wire from different producers and with different heat treatment conditions, for a wild comparison and study.

\section{REFERENCES}

[1] T. Isono, Y. Nunoya, and T. Ando, "Development of $10 \mathrm{kA}$ Bi2212 conductor for fusion application," IEEE Trans. Appl. Supercond., vol. 13, no. 2, pp. 1512-1515, Jun. 2003.

[2] X. F. Liu, J. X. Zheng, H. Wu, and S. S. Du, "Conceptual design and analysis of CFETR TF coil," J Fusion Energy, vol. 34, no. 5, pp. 10271032, Oct. 2015.

[3] H. Wada and T. Kiyoshi, "Development of $1 \mathrm{GHz}$ class NMR magnets," IEEE Trans. Appl. Supercond., vol. 12, no. 1, pp. 715-717, Mar. 2002.

[4] B. Tenhaken, H. H. J. Tenkate, and J. Tenbrink, "Compressive and tensile axial strain reduced critical currents in BI-2212 conductors," IEEE Trans. Appl. Supercond., vol. 5, no. 2, pp. 1298-1301, Jun. 1995.

[5] B. Tenhaken, A. Godeke, H. J. Schuver, and H. H. J. Tenkate, "Descriptive model for the critical current as a function of axial strain in Bi-2212/Ag wires," IEEE Trans. Magn., vol. 32, no. 4, pp. 2720-2723, Jul. 1996.

[6] B. Ten Haken, "Strain effects on the critical properties of high-field superconductors," Ph.D. dissertation, Faculty Sci. Technol. Univ. Twente, Enschede, The Netherlands, 1994.

[7] J. W. Ekin, D. K. Finnemore, Q. Li, J. Tenbrink, and W. Carter, "Effect of axial strain on the critical current of Ag-sheathed Bi-based superconductors in magnetic-fields up to 25-T," Appl. Phys. Lett., vol. 61, no. 7, pp. 858-860, Aug. 17, 1992.

[8] R. Bjoerstad et al., "Strain induced irreversible critical current degradation in highly dense Bi-2212 round wire," Supercond. Sci. Technol., vol. 28, no. 6, Jun. 2015, Art. no. 062002.

[9] B. Tenhaken, A. Godeke, and H. H. J. Tenkate, "The influence of compressive and tensile axial strain on the critical properties of $\mathrm{Nb}_{3} \mathrm{Sn}$ conductors," IEEE Trans. Appl. Supercond., vol. 5, no. 2, pp. 1909-1912, Jun. 1995.

[10] H. J. N. van Eck, D. C. van der Laan, M. Dhalle, B. ten Haken, and H. H. J. Ten Kate, "Critical current versus strain research at the University of Twente," Supercond. Sci. Technol., vol. 16, no. 9, pp. 1026-1030, Sep. 2003.

[11] M. Polak et al., "Current transfer lengths and the origin of linear components in the voltage-current curves of Ag-sheathed BSCCO components," Supercond. Sci. Technol., vol. 10, no. 10, pp. 769-777, Oct. 1997.

[12] D. M. J. Taylor and D. P. Hampshire, "The scaling law for the strain dependence of the critical current density in $\mathrm{Nb}_{3} \mathrm{Sn}$ superconducting wires," Supercond. Sci. Technol., vol. 18, no. 12, pp. S241-S252, Dec. 2005.

[13] A. Nijhuis and R. P. P. van Meerdervoort, "The effect of axial and transverse loading on the transport properties of ITER $\mathrm{Nb}_{3} \mathrm{Sn}$ strands," Supercond. Sci. Technol., vol. 26, no. 8, Aug. 2013, Art. no. 084004.

[14] A. Godeke, B. Ten Haken, H. H. J. Ten Kate, and D. C. Larbalestier, "A general scaling relation for the critical current density in $\mathrm{Nb}_{3} \mathrm{Sn}$," Supercond. Sci. Technol., vol. 19, no. 10, pp. R100-R116, Oct. 2006.

[15] X. F. Lu et al., "Electromechanical characterization of Bi-2212 strands," IEEE Trans. Appl. Supercond., vol. 21, no. 3, pp. 3086-3089, Jun. 2011.

[16] M. Sugano, K. Itoh, and T. Kiyoshi, "Strain dependence of critical current in Bi2212W \& $\mathrm{R}$ wires under magnetic field up to $30 \mathrm{~T}$," IEEE Trans. Appl. Supercond., vol. 16, no. 2, pp. 1039-1042, Jun. 2006.

[17] P. Li, Y. Wang, A. Godeke, L. Ye, G. Flanagan, and T. Shen, "Thermalmechanical properties of epoxy-impregnated Bi-2212/Ag composite," IEEE Trans. Appl. Supercond., vol. 25, no. 3, Jun. 2015, Art. no. 8400904.

[18] C. Dai, B. Liu, J. Qin, F. Liu, Y. Wu, and C. Zhou, "The axial tensile stress-strain characterization of Ag-sheathed Bi2212 round wire," IEEE Trans. Appl. Supercond., vol. 25, no. 3, Jun. 2015, Art. no. 6400304.

[19] M. Sugano and K. Osamura, "Stress-strain behavior and degradation of critical current of Bi2223 composite tapes," Physica C, vol. 402, no. 4, pp. 341-346, 2004.

[20] A. Godeke et al., "Critical current of dense Bi-2212 round wires as a function of axial strain," Supercond Sci. Technol., vol. 28, no. 3, 2015, Art. no. 032001.

[21] N. Cheggour et al., "Reversible effect of strain on transport critical current in $\mathrm{Bi} 2 \mathrm{Sr} 2 \mathrm{CaCu} 2 \mathrm{O} 8+\mathrm{x}$ superconducting wires: A modified descriptive strain model," Supercond. Sci. Technol., vol. 25, no. 1, 2012, Art. no. 015001 . 